\title{
Harlequin Ichthyosis in a Filipino Newborn: Management Pearls in a Resource-limited Setting
}

\author{
Jolene Kristine G. Gatmaitan-Dumlao, MD, ${ }^{1}$ Erin Jane L. Tababa, MD, ${ }^{2}$ \\ Cindy Jao-Tan, MD ${ }^{3,4}$ and Ma. Lorna F. Frez, MD ${ }^{1}$ \\ ${ }^{1}$ Department of Dermatology, Philippine General Hospital, University of the Philippines Manila \\ ${ }^{2}$ Healthserv Los Baños Medical Center, Laguna, Philippines \\ ${ }^{3}$ Manila Doctors Hospital, Manila, Philippines \\ ${ }^{4}$ Section of Dermatology, University of the East Ramon Magsaysay Medical Center, Manila, Philippines
}

\begin{abstract}
Introduction. Harlequin ichthyosis (HI) is a rare type of autosomal recessive congenital ichthyosis. There are approximately 200 documented cases worldwide, with less than five published reports in the Philippines. Despite its rarity, current literature suggests a better prognosis for these patients.

Case description. We describe a preterm male newborn who presented at birth enclosed in a thick hyperkeratotic armor-like scale plates with areas of fissures, with associated ectropion, conjunctiva dehiscence, and eclabium. The thickened encasement also covered the hands and feet, causing severe contractures. A diagnosis of harlequin ichthyosis was given based on the clinical features. The patient was managed through a multidisciplinary approach, including referral to the tele-ichthyosis platform of a US-based foundation for patients with ichthyosis. Thermoregulation, nutrition, and hydration were carefully managed. Bland emollients were applied generously following normal saline soaks to improve barrier protection. Acitretin was administered on day 2 of life to facilitate the desquamation of the thickened encasement. A marked decrease in erythema and the thickness of the hyperkeratotic skin, and reduced conjunctival dehiscence were noted after one week of therapy. However, the constrictions on the hands and feet showed bluish discoloration and signs of necrosis. Linear band excision was performed to release the constrictors. Despite aggressive management, the patient succumbed to sepsis on day 12 of life.
\end{abstract}

Conclusion. Improved prognosis amongst HI patients is correlated with optimal quality of care regardless of resource limitations. A multidisciplinary approach and early administration of retinoids cannot be overemphasized. Linear band excision within the first week of life is suggested for constrictions on the extremities that do not improve with retinoids to avoid necrosis and autoamputation.

Key Words: harlequin ichthyosis, acitretin, tele-ichthyosis

Presented (oral presentation) at the International Society of Dermatology (ISD) on February 4, 2017, Regional Meeting in Dubrovnik, Croatia. Scholarship and travel grant recipient.

Poster Presentation, Philippine General Hospital Research Forum 2016.

Poster presented at the Philippine Dermatological Society Annual Convention on November 3-5, 2016 in EDSA Shangri-La, Mandaluyong City, Philippines.

Corresponding author: Jolene Kristine G. Gatmaitan-Dumlao, MD Department of Dermatology Philippine General Hospital

University of the Philippines Manila

Taft Avenue, Manila 1000, Philippines

Notre Dame de Chartres Hospital

Baguio City 2600, Philippines

Email: jolenegatmaitan@gmail.com

\section{INTRODUCTION}

Harlequin ichthyosis (HI) is a rare, autosomal recessive condition, which presents at birth. This condition occurs in 1 in 300,000 births. ${ }^{1}$ Infant with this condition is born encased with a thick, hyperkeratotic plate-like covering that constitutes a significantly thickened stratum corneum and is associated with dysmorphic features, including ectropion and eclabium, and digital contractures. ${ }^{2}$

$\mathrm{HI}$, results from mutations of the ATP-binding cassette A12 gene, which codes for the ABCA12 lipid transporters in the lamellar granules. ${ }^{3,4}$ These lamellar granules transport essential lipids and lipid processing enzymes, proteases, and protease inhibitors from the cell to the intercellular space. The essential lipids contribute to the skin's efficient barrier, whereas the proteases act on the corneodesmosomes, which facilitate the desquamation of the corneocytes. With 
the mutation of the ABCA12 gene, there is impaired desquamation and barrier protection in the skin. ${ }^{5}$

Worldwide, there have been approximately 200 reported cases. The most comprehensive literature review was done in 2011 by Rajpopat et al., which included the patients' treatment course, outcomes, and associated gene mutations. Among the 45 cases reviewed, 25 (56\%) were survivors at the time of the study, ranging from 10 months to 25 years old. The mean gestational age at birth was 35 weeks. In the past, this condition was fatal in the newborn period. ${ }^{1}$ Prognosis of these patients has dramatically improved over the years, especially with a better quality of care and early administration of retinoids, ideally within seven days., Early introduction of oral retinoids was seen to improve survival. In the study, $84 \%$ of the patients who received oral retinoids survived. The overall mortality rate was $44 \%$, with the age of death ranging from day 1 to day 52 . The mortality rate among those who were not given oral retinoids was higher, wherein $76 \%$ died. Patients most commonly succumbed to sepsis, respiratory failure, or a combination. ${ }^{6}$

In the Philippines, a literature search using the HERDIN database showed three published case reports of HI. ${ }^{7-9}$ All of the patients were born pre-term to non-consanguineous parents. They were managed accordingly in the respective institutions but expired due to sepsis. ${ }^{7-9}$ Based on the Philippine Dermatological Society data registry, 11 patients with harlequin ichthyosis were seen at the dermatology clinics from 2011 to 2019 with a prevalence rate of $0.0017 \% .^{10}$

There is a lack of treatment guidelines due to the rarity of the disease. ${ }^{2}$ Hence, this report will focus on the management of the patient in a resource-limited setting. Emphasis is given on the importance of a multidisciplinary approach and early administration of retinoids. Updates and recommendations based on existing literature are also discussed.

\section{CASE REPORT}

A newborn male from Biñan, Laguna, was born preterm 33-34 weeks by late ultrasound to a 31-year-old G3P2 (gravida 3, para 2) (2002) via spontaneous vaginal delivery at a lying-in clinic by a midwife. The mother had two children from a previous partner, in which both pregnancies were unremarkable. The patient was firstborn to non-consanguineous parents, with no family history of keratinizing disorders.

The rest of the review of systems was unremarkable. Mother had a regular prenatal check-up by an obstetrician with adequate supplementation of multivitamins, ferrous sulfate, and folic acid. No abnormalities or illnesses were noted during the prenatal period. There was no exposure to teratogens and other chemicals. Ultrasound done at 29 weeks AOG (age of gestation) revealed single live uterine pregnancy, 28 4/7 weeks AOG, with anterior placenta Gr. 2, normohydramnios. Family medical history revealed no history of congenital anomalies, diabetes, hypertension, atopy, asthma, and other diseases. The patient was born to a 31-year-old mother who worked as a factory worker for an electronics company for about five years, while the father is a 31-year-old employee working as a distributor of diesel gas.

The patient had good pulses, heart rate, respiratory rate, and temperature for a pre-term baby on physical examination. The patient weighed 2700 grams. Head and neck examination revealed outward eversion of the lower eyelids (ectropion) with exposed bulbous erythematous, edematous protruding conjunctivae (chemosis), and a gaping, fish-like mouth, with pink mucosa (eclabium) (Figure 1). The nares were patent, with visibly flattened ala. The pinnae in both ears were underdeveloped, with the right side more developed than the left (Figure 2). The external auditory meatus was visible and looked patent.

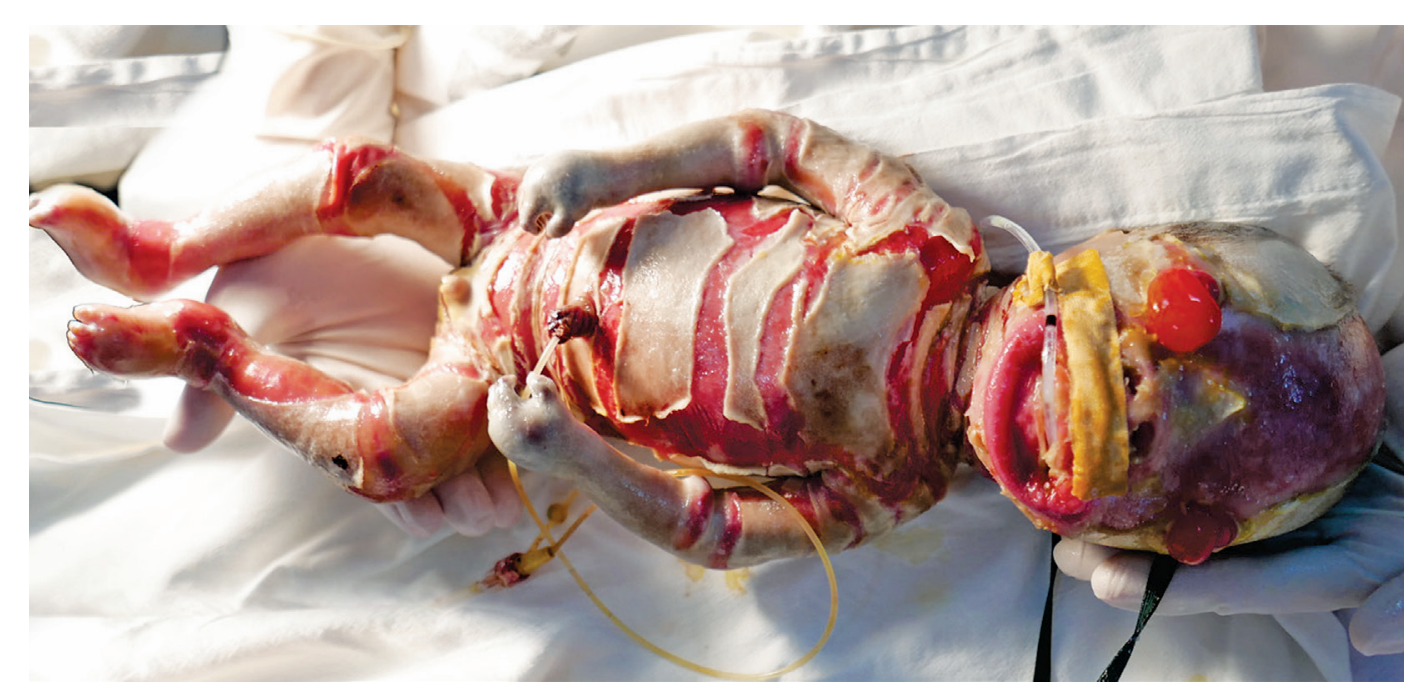

Figure 1. Newborn with thick hyperkeratotic plate-like encasement with fissures, upon presentation at the emergency department. 

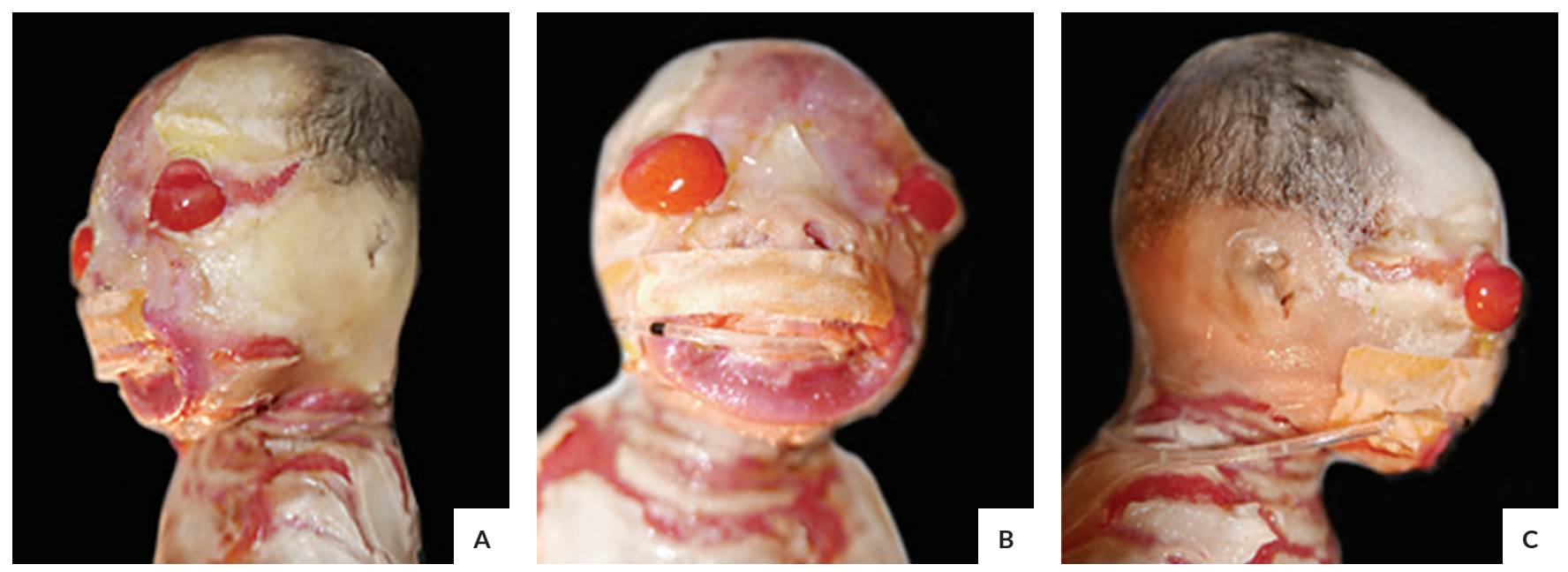

Figure 2. Facial profile of patient showing ectropion, conjunctival dehiscence with chemosis, patent nares, gaping mouth, ears covered thick, hyperkeratotic skin (A, B, C).
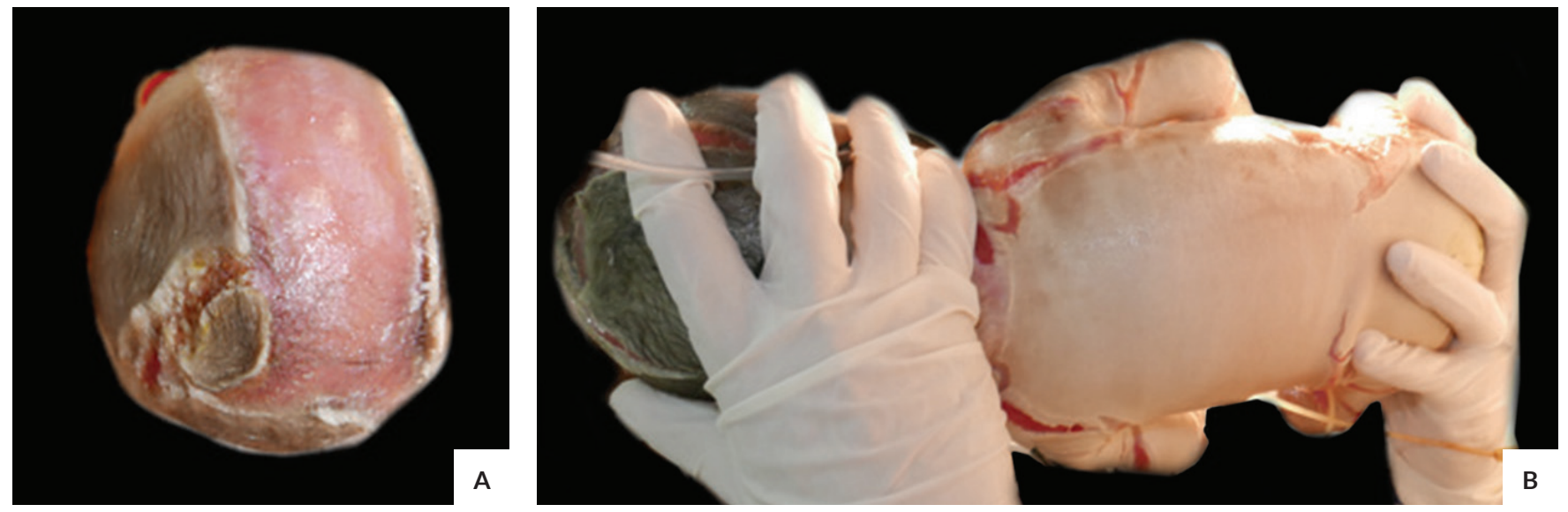

Figure 3. (A) There are fine black terminal hairs in the parieto-occipital area of the scalp, the left side covered with thick platelike stratum corneum with fissures. There are no signs of aplasia cutis. (B) The thickened skin on the back showed minimal fissures.

The patient was generally covered in a thick hyperkeratotic plate-like outer covering, thickened on the scalp, back, hands and feet. There were fine black terminal hairs in the parieto-occipital area of the scalp (Figure 3). There were no signs of aplasia cutis. The thickened skin on the trunk and extremities had fissures with underlying erythema. The fingers and toes were fully developed, with no noted syndactyly; however, the nails were not fully visualized because of the thick hyperkeratotic encasement. The thickened encasement also covered the hands and feet, which caused severe contractures. The chest, cardiac and abdominal findings were unremarkable.

\section{CLINICAL COURSE}

At the emergency room (ER), the pediatrics team stabilized the patient and provided supportive care. Umbi- lical catheterization was done, fluids were carefully managed, and adequate nutrition was ensured. The patient was referred to the dermatology service on day 1 of life. Emollients were prescribed, and laboratory tests, including liver function tests, blood urea nitrogen, creatinine, and lipid profile, were ordered in preparation for retinoid use. The patient was started on acitretin at $1 \mathrm{mg} / \mathrm{kg} / \mathrm{dose}$ on day 2 . Due to the unavailability of a humidified isolette, wet wraps with plain normal saline solution (0.9\%) and application of emollients were done for the patient three times a day. Bland emollients were applied as often as possible. Close monitoring of temperature, respiration, and fluid and electrolyte status was performed. A multidisciplinary approach was advocated with the following services onboard at the time of admission: general pediatrics, genetics, neonatal intensive care unit (NICU), otorhinolaryngology, pain service, and surgery. 
The dermatology service referred the patient to F.I.R.S.T. (Foundation for Ichthyosis and Related SkinTypes) via their tele-ichthyosis platform. This is a US-based organization that supports patients with ichthyosis and provides expert assistance to physicians and dermatologists. Unfortunately, despite adequate genetic counseling, the parents declined to undergo genetic testing under the F.I.R.S.T. foundation.

The ophthalmology service assessed the patient to have conjunctival dehiscence with non-visualization of the retina and prescribed artificial tears and carbomer eye gel to prevent dryness. The otorhinolaryngology service noted microtia and advised a CT scan to assess the extent of the facial abnormalities further. The pain service recommended fentanyl but eventually started paracetamol for pain management. Constrictions of the digits of the hands and feet from the thick encasement were also observed. The patient was given urea lotion for the constrictions, while the rehabilitation service advised a range of motion activities. At this point, the surgery service focused on the dressing of the wounds.

On day 9 of life, which corresponded to one week of treatment with acitretin, there was a marked decrease in erythema of the skin with thinning of the hyperkeratotic plate-like skin (Figure 4). The chemosis of the conjunctiva subsided and reduced tautness of the skin around the eyes and mouth. However, the constrictions on the hands and feet showed bluish discoloration of distal areas (Figure 5). The F.I.R.S.T. expert panel, through tele-ichthyosis consultation, suggested the continuation of acitretin and urea lotion. Topical tazarotene was also offered but was unavailable at the institution.

On day 10 of life, there was a note of persistence of the bluish discoloration with associated beginning maceration of the skin on both hands and foul-smelling discharge. Linear band excision, which entailed placing vertical incisions on the thickened encasement to release the constrictures, was then performed by the dermatology service upon the advice of the F.I.R.S.T. expert panel. ${ }^{11}$ Superficial incisions were done on bilateral feet to release the toes (Figure 6) without reaching the dermis. The thickened hyperkeratotic encasement's consistency was thick and wax-like during the release but was pliable afterward with note of the pinkish base. No bleeding was noted. The left heel was more hyperkeratotic compared with the right, with the consistency of a thick callous. The skin on both hands was macerated and necrotic; hence, the incisions were not done. Gentle debridement using gauze was advised after plain normal saline wash irrigation. Referral back to the surgery service was suggested for possible debridement and to inquire if amputation should be considered for source control.

On day 11 of life, the patient developed abdominal enlargement, with associated pallor on the perioral area. There was also note of worsening necrosis on bilateral hands with associated foul-smelling discharge. Blood cultures showed Klebsiella pneumoniae, which was sensitive

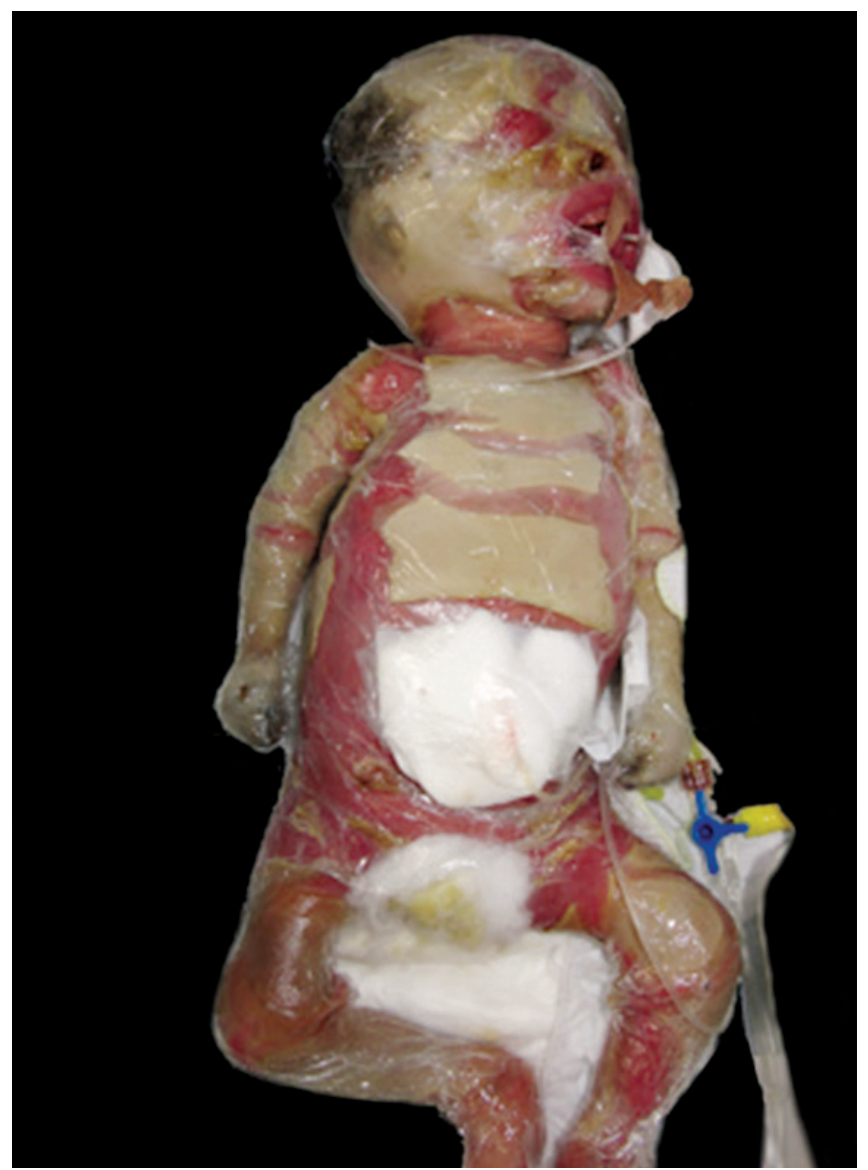

Figure 4. Patient at day 8 on acitretin. Note the decrease in conjunctival swelling, thinning of the edges of the thick plates, and the decrease in underlying erythema.

to colistin and cotrimoxazole. Ascites was noted on x-rays, but holoabdominal ultrasound showed normal liver. On day 12 , the patient succumbed to sepsis.

\section{DISCUSSION}

\section{Diagnostics}

Harlequin ichthyosis is usually diagnosed clinically. In the rare event that biopsy was done, the histopathologic findings include marked hyperkeratosis and hypogranulosis, with the stratum corneum ten times thicker than the spinous layer; the dermis is essentially normal.,12 Prenatal assessment may be done for suspicious HI phenotypes via amniotic sampling at 17 weeks. Also, prenatal 2D or 3D ultrasonography (UTZ) commonly shows a gaping mouth and bulging eyes, which are features of HI. ${ }^{9,12}$ The UTZ for the case patient done at 29 weeks revealed normal findings.

Monitoring for infection and electrolyte abnormalities is essential, especially in the early stages. ${ }^{2}$ Laboratory tests in preparation for the use of retinoids are also requested, such as liver function tests, blood urea nitrogen, creatinine, and lipid profile. 

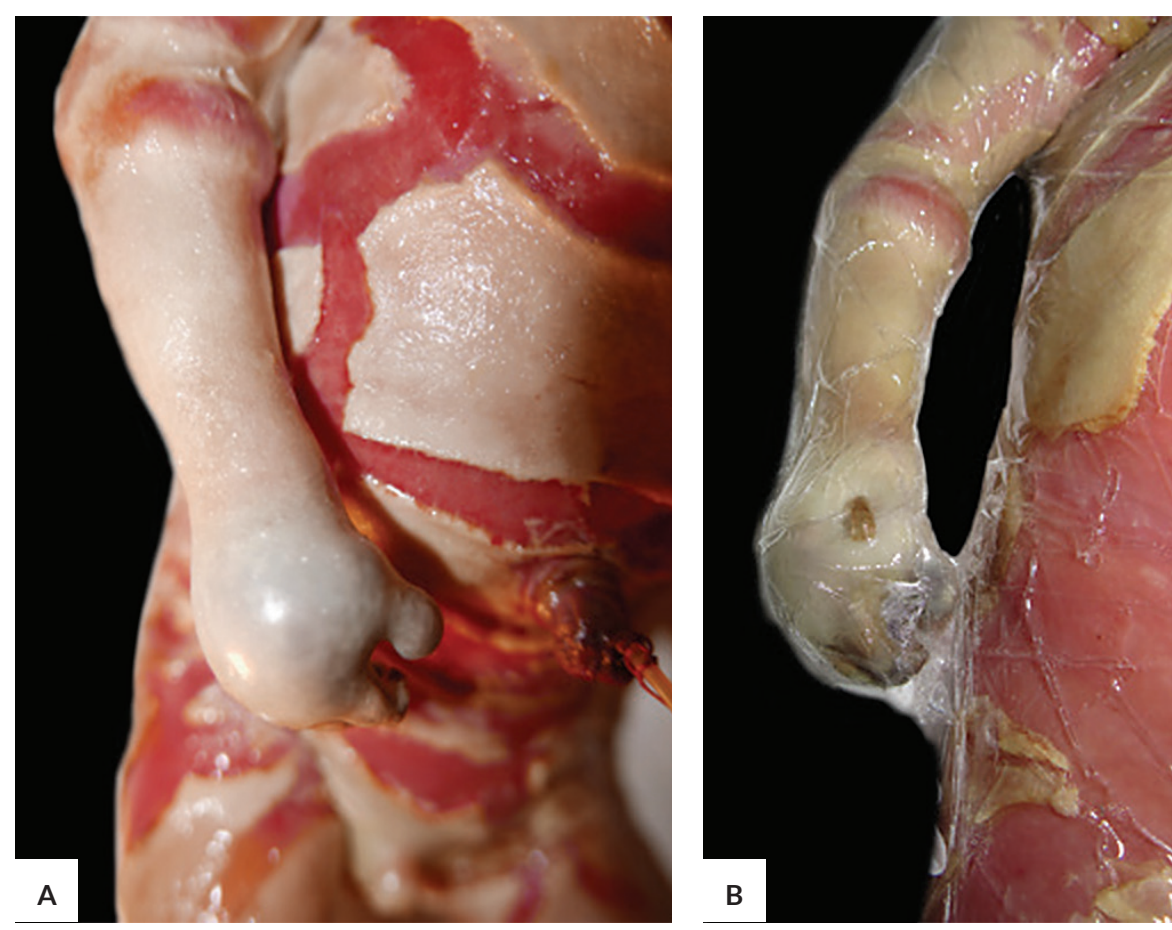

Figure 5. (A) The fingers were covered with thickened plate-like encasement with noted tautness seen on day 1 of life. (B) Decrease in erythema of the skin and thinning of the encasement after 8 days of acitretin. There was beginning bluish discoloration of fingertips.

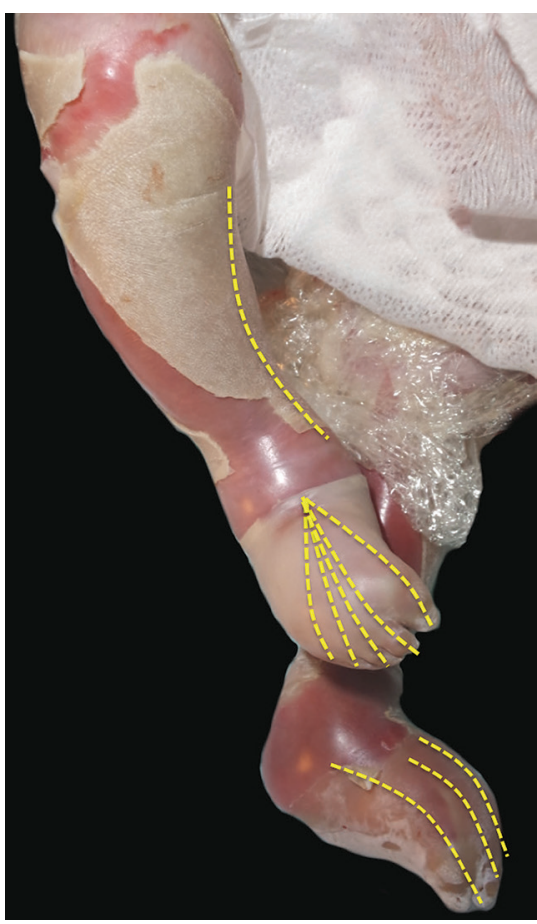

Figure 6. Placement of superficial incisions in the linear band excision performed on the legs and feet of the patient.

\section{Management of the hyperkeratotic plate-like stratum corneum}

Early initiation of retinoids has significantly improved HI patients' prognosis, and administration is ideally done within the first three days of life. ${ }^{6}$ Retinoids, which are natural or synthetic derivatives of vitamin A or retinol, are mainly acquired through diet, absorbed within the intestinal lumen, and stored mostly in the liver. Retinoids help induce normal differentiation of cells through actions in the DNA nuclear receptors found in the skin, namely the retinoic acid receptor (RAR) and retinoid $x$ receptor $(\mathrm{RXR}) .{ }^{13}$

Among 45 cases of harlequin ichthyosis reviewed in 2011, twenty-four patients were given retinoids within the first week of life, and 20 patients (83\%) survived. ${ }^{6}$ Twenty patients received acitretin, while two patients received etretinate, and two patients received isotretinoin. When the study was performed, the age range of harlequin ichthyosis survivors was ten months to 25 years old. This was in contrast to the mortality rate of $76 \%$ in the patients not given retinoids (16 patients out of 21 patients). ${ }^{6}$ Retinoids facilitate the shedding of the thickened hyperkeratotic encasement and promote phenotypic shift, usually to congenital ichthyosiform erythroderma (CIE). However, aggressive thermoregulation, nutrition, and hydration, and application of bland emollients are still highly recommended with retinoid therapy. ${ }^{6}$ Improved neonatal care and early introduction of oral retinoids contribute significantly to the survival advantage..$^{2,6,14}$ The patient in this study was given acitretin on day 2 of life and was monitored closely along with the other managing services.

The thick plate-like scales lead to considerable restriction of motion and may affect essential functions. Truncal fissures may impair deep breathing, thereby increasing the risk for pulmonary complications. ${ }^{5}$ Pain management must also be ensured. Restricted mobility and arrested development of vital structures may lead to the appearance of the characteristic dysmorphic features seen in HI, such as ectropion, conjunctival dehiscence, eclabium, and hypoplastic ears. ${ }^{2}$ These clinical features were previously described to improve with administration of retinoids, gentle and meticulous debridement, and application of moisturizers. ${ }^{15}$ For severe ectropion, surgery may be needed to avoid complications such as blindness and deprivation amblyopia. ${ }^{15,16} \mathrm{~A}$ facial CT-scan may prove useful to rule out other bony and soft tissue abnormalities, such as choanal atresia, ear abnormalities such as microtia, and other craniofacial pathologies. ${ }^{8}$

Constrictions of the digits of the hands and feet from the thick hyperkeratotic encasement were also observed in our patient. Autoamputation and compartment syndrome pose a threat to the newborn and warrants medical attention. ${ }^{11,17}$ Prolonged constrictions of digits due to the presence of thick hyperkeratotic encasement was previously described. ${ }^{11}$ In a case report of a two-month-old patient with lamellar ichthyosis affecting the third and fourth digits of the left 
hand, the dermatologist performed surgical release of the constrictions using surgical scalpel blade no. 11 on the left palmar aspect, which led to the resolution of the presenting erythema and edema after one week. ${ }^{17}$ A similar approach was performed in a newborn with harlequin ichthyosis, who presented with constrictions on the limbs, digits, and toes at day 3 of life while on acitretin therapy. An escharotomylike procedure was performed at the bedside by a plastic surgeon and a pediatric surgeon, termed as linear band excision. Incisions were done longitudinally, oriented toward the tendon pathways. Straight incision lines were placed over the dorsal and ventral aspects of the forearms and calves and the ventral hands and feet. The patient was able to improve the range of motion of all extremities after one week. ${ }^{11}$ The surgical procedure should be done at the earliest sign of tissue discoloration to prevent compartment syndrome and autoamputation. Local anesthesia (lidocaine $0.25 \%$ with epinephrine) was adequate. Post-procedure care was also emphasized, with the use of antibiotic ointment and petrolatum gauze. ${ }^{18}$

For the patient, in this case, the retinoids significantly improved the erythema of the skin. There was also visible thinning of the thickened encasement, especially towards the edges of the plate-like stratum corneum in between the fissures. There were noted improvement in the mobility of the feet of the after linear band incision and gentle debridement. However, worsening necrosis of the hands prompted follow-up surgery referral for possible surgical debridement and source control.

\section{Management of the skin impairment}

The skin's normal functions are maintaining a barrier, preventing dehydration, decreasing the risk for systemic absorption, maintaining thermoregulation, addressing interpersonal communication, and avoiding infection. For patients with HI, problems will arise due to the skin impairment, subsequently undermining these normal functions. Hence, another therapeutic goal is to relieve the symptoms brought about by the skin impairment. ${ }^{1}$

Bland emollients should be applied to the patient generously to provide protection and to prevent transepidermal cutaneous losses. ${ }^{2}$ Topical treatments should be carefully monitored because of the increased risk of systemic absorption. In particular, the use of silver-impregnated ointments was not recommended by the F.I.R.S.T. expert panel due to the risk of toxicity. Antibiotic creams, such as bacitracin, may be used in addition to the wound care in case the surgical release is performed for the constrictions. ${ }^{18}$

Nutrition and hydration should be carefully managed. In an ideal setting, the use of $60-70 \%$ humidified isolettes is recommended with designated "off" times for breastfeeding and bonding with parents. ${ }^{19}$

For this patient, he was given petroleum jelly with occlusion, fluids were carefully balanced and adjusted by the general pediatrics service, with expressed breast milk given incrementally per day. However, the patient was admitted to the wards instead of the pediatric intensive care unit or protective isolation due to lack of available beds.

Despite the intimidating appearance, skin-to-skin contact and breastfeeding are still recommended. Genetic counseling is also essential. The patient was referred to F.I.R.S.T. for genetic testing at no cost to both the child and the parents. Unfortunately, the father did not consent to the gene testing.

\section{Infection Control}

Sepsis remains a constant threat in the first month, while the fissures and hyperkeratotic collodion membrane are shedding. Thus, close monitoring, ideally in a NICU setting, with strict sepsis precautions should be advocated. Aggressive treatment of bacterial and fungal infections should be done. There is no evidence for prophylactic antibiotic and antifungal use ${ }^{2}$, since these may favor the growth of Pseudomonas aeruginosa and Candida. Our patient was given ampicillin and amikacin from the time of ER admission, which was subsequently shifted to vancomycin and meropenem due to the appearance of Klebsiella sp. on blood culture. Cardiorespiratory distress and sepsis remain to be the primary causes of mortality of patients with $\mathrm{HI}$ as seen in our patient. ${ }^{6-9}$

\section{CONCLUSION}

Harlequin ichthyosis remains to be best managed using a multidisciplinary approach. Despite being in a resource-limited setting, good quality of care is advocated, especially with the improved prognosis of patients with HI worldwide. Greater awareness of the clinical features and clinical course and the early administration of acitretin should be emphasized. Linear band excision within the first week of life is suggested for constrictions on the extremities that do not improve with retinoids to avoid necrosis and autoamputation.

\section{Acknowledgments}

The authors would like to express their gratitude to the Tele-ichthyosis team of the Foundation for Ichthyosis and Related Skin Types (F.I.R.S.T.) for their expert opinion on the management of the patient at the time of admission.

\section{Statement of Authorship}

All authors participated in data collection and analysis, and approved the final version submitted.

\section{Author Disclosure}

All authors declared no conflicts of interest.

\section{Funding Source}

This paper was self-funded. 


\section{REFERENCES}

1. Oji V, Tadini G, Akiyama M, Bardon CB, Bodemer C, Bourrat E, et al. Revised nomenclature and classification of inherited ichthyoses: Results of the First Ichthyosis Consensus Conference in Soreze 2009. J Am Acad Dermatol. 2010 October;63(4):607-41.

2. Harvey HB, Shaw MG, Morrell DS. Perinatal management of harlequin ichthyosis: a case report and literature review. J Perinatol. 2010;30(1):66-72.

3. Akiyama M. Pathomechanisms of harlequin ichthyosis and $\mathrm{ABCA}$ transporters in human diseases. Arch Dermatol. 2006;142(7):914-8.

4. Akiyama M. Updated molecular genetics and pathogenesis of ichthyosis. Nagoya J Med Sci. 2011 August;73(3-4):79-90. Pubmed PMID 21928690; Pubmed Central PMCID: PMC4831217.

5. Hovnanian A. Harlequin ichthyosis unmasked: a defect of lipid transport. J Clin Invest. 2005 July;115(7):1708-10.

6. Rajpopat S, Moss C, Mellerio J, et al. Harlequin Ichthyosis. Arch Dermatol. 2011 June;147(6):681-6.

7. Kho J, Santos-Belisario M. Harlequin Ichthyosis - a case report. J Philipp Dermatological Soc. 1998;7(2):85-90.

8. Oconer J, Portugal R, Almazan-Aguilar N, Lapena J. Harlequin Ichthyosis. Philipp J Otorhinolaryngol Head Neck Surg. 2004; 19(3):3-4.

9. Laksamana O, Bernardino M, Santos R, Castrillo J. A Case of Harlequin Ichthyosis. Philipp J Obstet Gynecol. 2011;35(2):83-7.

10. Philippine Dermatological Society Health Information Systems. Philippine Dermatological Society. c2011 [updated (March 2019); cited 2019 December)]. Available from: pdshis@outlook.com
11. Tontchev G, Silverberg NB, Shlasko E, Henry C, Roberts JL, Roth MZ. Techniques for toddlers: Linear band incision for harlequin ichthyosis with associated compartment syndrome. Pediatr Dermatol. 2014;31(5):625-9.

12. Rathore S, David L, Beck M, Bindra M, Arunachal G. Harlequin Ichthyosis: Prenatal Diagnosis of a Rare Yet Severe Genetic Dermatosis. J Clin Diagnostic Res. 2015;9(11):11-3.

13. Thielen AM, Saurat JH. Retinoids. In Bolognia, JL; Jorizzo JSJ editors. Dermatology. 3rd ed. Elsevier Inc; 2012. p 2089-103.

14. Dubey AK, Tuibeqa IV, Pio NB, Dubey AK, Tuibeqa IV, Pio NB. Harlequin ichthyosis: A rare dermatological disorder Harlequin ichthyosis: A rare dermatological disorder. Int J Case Rep Images. 2014;5(8):590-4.

15. Hazuku T, Yamada K, Imaizumi M, et al. Unusual protrusion of conjunctiva in two neonates with Harlequin ichthyosis. Case Rep Ophthalmol. 2011;2(1):73-7.

16. Kün-Darbois JD, Molin A, Jeanne-Pasquier C, Paré A, Bénateau $\mathrm{H}$, Veyssière A. Facial features in Harlequin ichthyosis: Clinical findings about 4 cases. Rev Stomatol Chir Maxillofac Chir Orale. 2016;117(1):51-3.

17. Roberts JB, Adelson D. Case report: Prolonged collodion membrane causing constrictive bands of the digits and treatment. Dermatol Online J. 2010;16(1):5-6.

18. Pet MA, Gupta D, Tse RW. Harlequin Ichthyosis: A Surgical Perspective. Pediatr Dermatol. 2016;33(5).

19. Nguyen MA, Gelman A, Norton SA. Practical Events in the Management of a Collodion Baby. JAMA dermatology. 2015;151(9):1031-2. 